\title{
Comparison between Certain Parameters for Diagnosis of Acute Myocardial Infarction
}

\author{
Muthear N. Dawood*
}

Akram J. Ahmed **

\begin{abstract}
Background and Objective: The identification and diagnosis of acute myocardial infarction (AMI) in patients presented with chest pain pose significant challenges, as the rate of missed AMI cases among patients who present to the emergency department remain inappropriately high. The aim of this work was to study the performance of three cardiac biomarkers, these are heart-type fatty acid binding protein (HFABP), troponin I ( $3^{\text {rd }}$ generation TOSOH AIA pack-assay) and myoglobin, for early identification of acute myocardial infarction.

Material and Methods: This study was designed as a case -control study. Participants were divided into two groups, the first one included 82 patients diagnosed to have AMI and the second group included 50 apparently healthy control subjects. Troponin I and myoglobin were measured by AIA-Pack assays from TOSOH bioscience while H-FABP was measured by ELISA technique.

Results: The area under the curve (AUC) for troponin I was the highest between the tested biomarkers $(0.972 \pm 0.012)$ compared with H-FABP and myoglobin which were found to be $(0.968 \pm 0.013)$ and $(0.921 \pm 0.022)$ respectively. Furthermore, by using the optimal cut-off values of the tested biomarkers, the sensitivity of H-FABP was the highest for those patients presented within the first six hours of the onset of chest pain (90.6\%), followed by troponion I and myoglobin $(81.3 \%$ and $78.1 \%$ respectively). From the other hand, the sensitivity of troponin I was the highest (98\%) for those patients presented after the sixth hour up to 12 hours from the onset of chest pain compared by H-FABP and myoglobin $(90 \%$ and $78 \%$ respectively).

Conclusion: The present study demonstrates that both improved sensitivity troponin I assay and H-FABP can provide valuable information for the diagnosis of AMI. However, for those patients who presented within the first 6 hours of the onset of chest pain, H-FABP will provide superior sensitivity, as application of an early biomarker potentially reduces diagnostic uncertainty for patients with suspected AMI that allow an earlier initiation of treatment, while for those patients who presented later after the $6^{\text {th }}$ hour up to 12 hours of the onset of chest pain, it is more appropriate to use troponin I assay as it provide higher sensitivity.
\end{abstract}

Key words: Acute Myocardial Infarction, Heart-type fatty acid binding protein, Myoglobin \&Troponin I.

\section{INTRODUCTION}

Chest pain is a common presentation to the emergency department and accounts for approximately $5-10 \%$ of all visits (Owens et al, 2010). The identification and diagnosis of acute myocardial infarction in these patients pose a significant challenges, as the rate of missed AMI cases among patients who present to the emergency department remain inappropriately high (Lin et al, 2012). Quick and thorough assessment of the patients will help in the accurate diagnosis and aid in early stratification which is essential for guiding the treatment (Kumar and Cannon, 2009). Whereas any delays in diagnosis of AMI or "ruling in" holds back prompt treatment. Furthermore, delays in excluding other heart diseases or "ruling out" will interfere with the evaluation of alternative diagnosis (Reiter et al, 2011). Besides the clinical history and the physical examinations of the suspected patients with AMI, ECG and cardiac biomarkers determinations are required for the proper assessment and diagnosis of the patients (Achar et al, 2005). ECG is very important tool in the diagnosis as it can monitors cardiac function in real time (Paterson and Herring, 2006). However, ECG alone is not always sufficient to diagnose AMI because ECG changes are absent in many patients and because ST segment deviation is observed in many other

* Assistant lecturer/Department of clinical laboratory sciences/College of Pharmacy/ University of Mosul/muthear@yahoo.com.

** Professor of chemical pathology/ Department of Biochemistry/ College of Medicine/ University of Mosul/ Akrami1948@vahoo.com. 
conditions (Achar et al, 2005). Therefore cardiac markers are important in the diagnosis of AMI (Mad et al, 2007). The ideal marker should quickly identify high risk patients for fast track treatment while excluding patients who are at low risk of life threatening diseases (Erhardt et $a l, 2002)$.

Cardiac troponins fulfill these criteria to a large extent, their high sensitivity for minor myocardial injury and almost total specificity to the cardiac muscle have made it possible as the preferred biochemical marker for the diagnosis of acute myocardial infarction (Jaffe et al, 2000) and nowadays cardiac troponins (troponin I and troponin T) have a central role in the diagnosis and management of patients with suspected acute coronary syndrome (Melanson et al, 2007). Other available biomarkers such as CKMB, myoglobin, NT-pro BNP or CRP did not provide superior clinical information over cardiac troponins (Eggers, 2007).

Recently a number of novel markers have been identified to predict outcome following an acute myocardial infarction or acute coronary syndrome for example heart-type fatty acid binding protein (H-FABP) (Chan and Ng, 2010, 34). However, despite the availability of variety of emerging novel biomarkers, the understanding for the roles and biochemistry of these various biomarkers in evaluating the disease process is still fairly limited and require more efforts (Das, 2012).

Besides that, there are continuous efforts to improve troponin assays by introducing a new generation of sensitive assays for cardiac troponins, this cadre of sensitive troponin assays might further enhance the accuracy of diagnosis of acute coronary syndrome (ACS) and therefore improve diagnostic sensitivity and specificity even in patients presenting early after the onset of chest pain (Keller et al, 2009).

\section{MATERIALS AND METHOD}

This study was carried out at the emergency department of Aljumhuri teaching hospital and the coronary care unit of Ibn Sina teaching hospital in Mosul city from March 2012 to February 2013, the study received the agreement of the Ethical Committee of Nineveh Governorate Health Department and approval of Mosul College of medicine- Postgraduate Studies Committee.

This study was designed as a case control study, participants was divided into two groups, the first one included 82 patients with mean age of $59.3 \pm 11.1$ years; $70.7 \%$ were males and $29.3 \%$ were females diagnosed to have AMI and the second group included 50 apparently healthy subjects with mean age of $55.3 \pm 7.8$ years; $62 \%$ were males and $38 \%$ were females as a control.

Following classical clinical presentation of acute myocardial infarction like ischemic chest pain, suspected patients initially attend the emergency department for initial assessment then were assigned to the coronary care unit (CCU) for the confirmation of the diagnosis with the help of senior physicians of cardiology.

All patients included in this study were admitted to the emergency department within 12 hours after the onset of chest pain. Patients were diagnosed to have AMI by the presence of classical chest pain $>20$ minutes, with characteristic ECG changes like pathological Q wave, elevation or depression of ST segment and/ or $\mathrm{T}$ wave changes. Furthermore, to confirm the diagnosis, all patients had shown a positive troponin I results which was tested between (12-24) hours from the onset of chest pain using point of care strips which has comparable diagnostic performance to the utilized quantitative testing strategy for AMI (Al-Mukhtar and Ahmad, 2011).

\section{Exclusion criteria:}

The criteria of exclusion were more than 12 hours after the onset of symptoms, neoplastic diseases, renal failure, myopathy and left bundle branch block.

\section{Study protocol:}

Blood samples were collected from each patient as soon as possible after admission, in gel separator tubes, using a disposable needle and syringe. Blood allowed to clot and then serum was separated by centrifugation at 3000 rpm for 10 minutes. The serum samples were divided into aliquots, coded and stored at $-20^{\circ} \mathrm{c}$. Another blood sample was collected from each patient after (12-24) hours from the onset of chest pain for immediate test of Troponin I by qualitative point of care testing, this test was intended to confirm the diagnosis of AMI and not for statistical reasons.

\section{Biochemical analysis:}

Point of care test strips were used from a kit supplied from Plasmatec laboratory product. A negative result indicated when the positive band in the strip remained pale while the control band turned pink-red. A positive result was indicated when both the positive and control bands turned pink-red.

Troponin I was measured using the thirdgeneration AIA-Pack assay for cTnI from 
TOSOH Corporation, Tokyo, Japan. Heart-type fatty acid binding protein (H-FABP) was measured using ELISA kit supplied by TSZ ELISA, USA. Myoglobin was measured using AIA-Pack assay for myoglobin from TOSOH bioscience, Tokyo, Japan.

\section{Statistic analysis:}

Statistical evaluation was performed using MedCalc software (version 12.7.2.0). Data were expressed as mean \pm S.E. Student's t- test was used to compare between continuous variables while chi- square was used to compare between categorical variables, receiver operator characteristic (ROC) curve was used to find AUC, optimal cut-off value, sensitivity and specificity for each marker. $P$ values $<0.05$ are considered statistically significant.

\section{RESULTS}

The baseline characteristics of the subjects in the studied groups are summarized in table 1 . The analysis of all measured biomarkers (troponin I, H-FABP and myoglobin) was showed significant differences between patients and control group ( $\mathrm{P}<0.0001$ Value) (table 2). Furthermore, the AUC was found to be the highest with Troponin I $(0.972 \pm 0.012)$ followed by H-FABP $(0.968 \pm 0.013)$ which was slightly lower than Troponin I, while myoglobin $(0.921 \pm 0.022)$ had the lowest AUC among these 3 markers (table 3). Further analysis of the data showed that Troponin I had the highest sensitivity, specificity and accuracy for discriminating AMI from healthy controls (table 4). Furthermore, AMI patients were divided into two subgroups, the first one included those patients who were admitted to the hospital within the first 6 hours from the onset of chest pain $(n=32)$ and the second subgroup included those who were admitted to the hospital after the $6^{\text {th }} \mathrm{h}$ up to 12 hours from the onset of chest pain $(\mathrm{n}=50)$. Using the optimal cut-off value that was obtained for each biomarker as described previously and as shown in table (4), the sensitivity of cardiac troponin I was increased from only $81.3 \%$ in subgroup 1 (patients presented within the first 6 hours from the onset of chest pain) to $98 \%$ in subgroup 2 (patients admitted to the hospital after the $6^{\text {th }}$ hour of chest pain up to 12 hours). On the other hand, HFABP showed comparable results of sensitivity for each subgroup of patients, the sensitivity was $90.6 \%$ in subgroup 1 and $90 \%$ in subgroup 2 . Myoglobin sensitivity also showed comparable results of sensitivity in both subgroups of patients, $78.1 \%$ in subgroup 1 and $78 \%$ in subgroup 2 (table 5).

Table 1: Baseline characteristics of the participants.

\begin{tabular}{|c|c|c|c|}
\hline \multicolumn{2}{|c|}{ Variables } & $\begin{array}{l}\text { Control } \\
\text { No. }(\%)\end{array}$ & $\begin{array}{c}\text { MI } \\
\text { No. }(\%)\end{array}$ \\
\hline \multicolumn{4}{|c|}{ Age group (years) } \\
\hline & $40-49$ & $16(32 \%)$ & $18(22.0 \%)$ \\
\hline & $50-59$ & $21(42 \%)$ & $22(26.8 \%)$ \\
\hline & $60-69$ & $12(24 \%)$ & $24(29.3 \%)$ \\
\hline & $70-79$ & $1(2 \%)$ & $12(14.6 \%)$ \\
\hline & $80-89$ & - & $6(7.3 \%)$ \\
\hline & Range of age & $42-72$ & $40-86$ \\
\hline & Mean \pm SD & $55.3 \pm 7.8$ & $59.3 \pm 11.1$ \\
\hline \multicolumn{4}{|l|}{ Gender } \\
\hline & Males & $31(62 \%)$ & $58(70.7 \%)$ \\
\hline & Females & $19(38 \%)$ & $24(29.3 \%)$ \\
\hline \multicolumn{4}{|l|}{ Residence } \\
\hline & Urban & $39(78 \%)$ & $52(63.4 \%)$ \\
\hline & Rural & $11(22 \%)$ & $30(36.6 \%)$ \\
\hline \multicolumn{4}{|c|}{ Body mass index (BMI) kg/m² } \\
\hline & Normal (18.5-25) & $22(44 \%)$ & $36(43.9 \%)$ \\
\hline & Dverweight (25.1-30) & $21(42 \%)$ & $20(24.4 \%)$ \\
\hline & Obese $(>30)$ & $7(14 \%)$ & $26(31.7 \%)$ \\
\hline
\end{tabular}


Table 2: The difference in mean $\pm \mathrm{SE}$, minimum and maximum values of studied biomarkers among controls and patients.

\begin{tabular}{|c|c|c|c|}
\hline Parameters & $\begin{array}{c}\text { Control } \\
\text { Mean } \pm \text { SE } \\
\text { Min-max }\end{array}$ & $\begin{array}{c}\text { Myocardial infarction } \\
\text { Mean } \pm \text { SE } \\
\text { Min-max }\end{array}$ & p-value \\
\hline $\begin{array}{c}\text { Troponin I } \\
(\mathrm{ng} / \mathrm{ml})\end{array}$ & $\begin{array}{c}0.024 \pm 0.002 \\
(0.00-0.06)\end{array}$ & $\begin{array}{c}17.42 \pm 2.08 \\
(0.02-60)\end{array}$ & $<0.0001$ \\
\hline $\begin{array}{c}\mathrm{H}-\mathrm{FABP} \\
(\mathrm{ng} / \mathrm{ml})\end{array}$ & $\begin{array}{c}8.97 \pm 0.6 \\
(3.2-18.4)\end{array}$ & $\begin{array}{c}27.3 \pm 1.2 \\
(6.4-63.8)\end{array}$ & $<0.0001$ \\
\hline $\begin{array}{c}\text { Myoglobin } \\
(\mathrm{ng} / \mathrm{ml})\end{array}$ & $\begin{array}{c}38.7 \pm 2.8 \\
(12.2-88.5)\end{array}$ & $\begin{array}{c}176.3 \pm 13.8 \\
(20.1-623.1)\end{array}$ & $<0.0001$ \\
\hline
\end{tabular}

Table 3: AUC for the measured parameters for the discriminating of subjects of MI from controls when presented within 12 hours from the onset of chest pain.

\begin{tabular}{|c|c|c|c|}
\hline Parameters & AUC \pm SE & $\mathbf{9 5 \%}$ CI of AUC & $\boldsymbol{P}$-value \\
\hline Troponin I & $0.972 \pm 0.012$ & $0.927-0.993$ & $<0.0001$ \\
\hline H-FABP & $0.968 \pm 0.013$ & $0.922-0.991$ & $<0.0001$ \\
\hline Myoglobin & $0.921 \pm 0.022$ & $0.862-0.961$ & $<0.0001$ \\
\hline
\end{tabular}

Table 4: Optimal cut-off value, the sensitivity, the specificity and the accuracy for the measured parameters for the discriminating of subjects of MI from controls when presented within 12 hours from the onset of chest pain.

\begin{tabular}{|l|c|c|c|c|}
\hline \multicolumn{1}{|c|}{ Parameters } & Optimal cut-off (ng /ml) & Sensitivity & Specificit & Accuracy \\
\hline Troponin I & $>0.05$ & $91.5 \%$ & $98 \%$ & $93.9 \%$ \\
\hline H-FABP & $>16.4$ & $90.2 \%$ & $96 \%$ & $92.4 \%$ \\
\hline Myoglobin & $>68.2$ & $78.1 \%$ & $92 \%$ & $83.3 \%$ \\
\hline
\end{tabular}

Table 5: Sensitivity of biomarkers in respect to time for discriminating patients with AMI from healthy control. Subgroup 1 included patients who presented within the first hours of the onset of chest pain and subgroup 2 included patients who presented after the $6^{\text {th }}$ hour up to 12 hours of the onset of chest pain.

\begin{tabular}{|c|c|c|c|}
\hline Parameter & $\begin{array}{c}\text { Optimal cut-off } \\
\text { value }\end{array}$ & $\begin{array}{c}\text { Sensitivity of } \\
\text { subgroup 1 }\end{array}$ & $\begin{array}{c}\text { Sensitivity } \\
\text { of subgroup }\end{array}$ \\
\hline Troponin I & $>0.05 \mathrm{ng} / \mathrm{ml}$ & $81.3 \%$ & $98 \%$ \\
\hline H-FABP & $>16.4 \mathrm{ng} / \mathrm{ml}$ & $90.6 \%$ & $90 \%$ \\
\hline Myoglobin & $>68.2 \mathrm{ng} / \mathrm{ml}$ & $78.1 \%$ & $78 \%$ \\
\hline
\end{tabular}

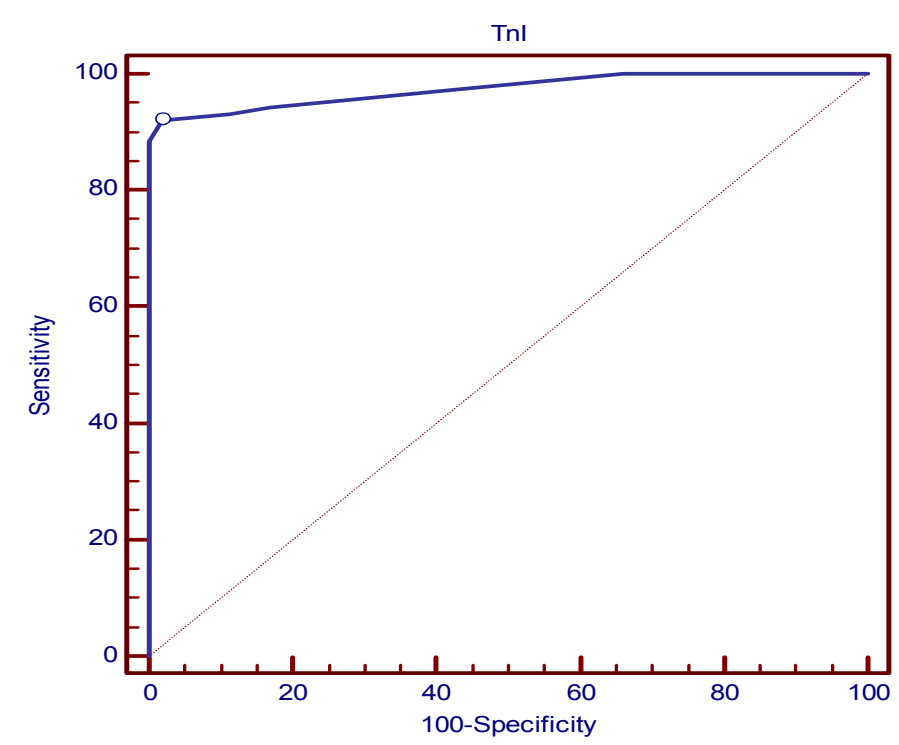

Figure 1: Troponin I ROC curve for discriminating patients who presented within 12 hours of the onset of chest pain from healthy subjects. 


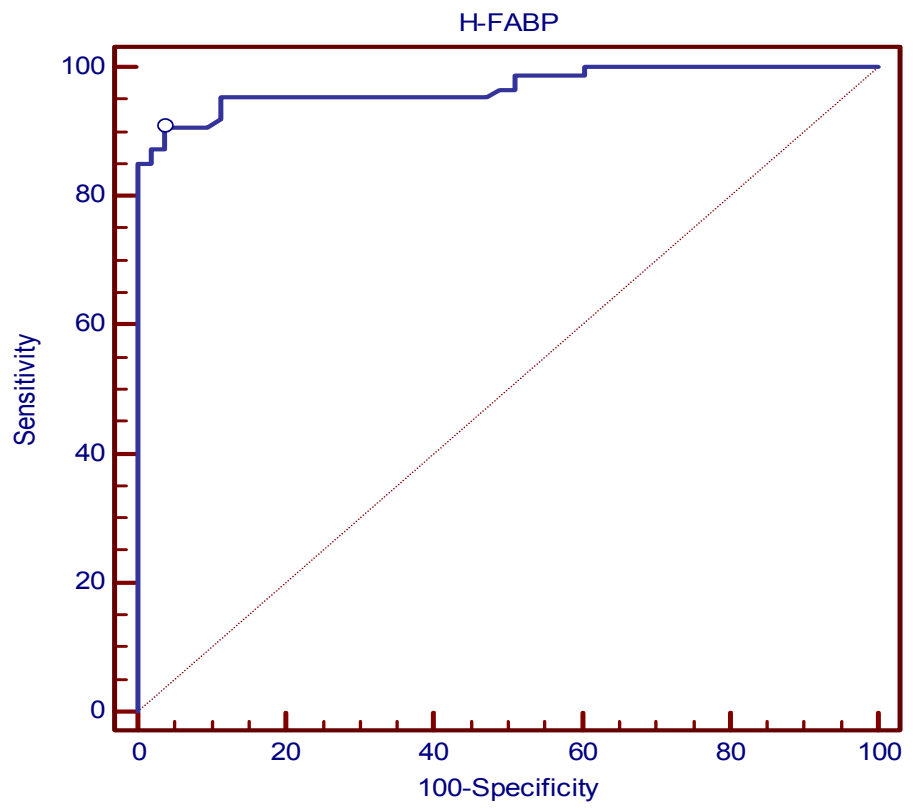

Figure2: H-FABP ROC curve for discriminating patients who presented within 12 hours of the onset of chest pain from healthy subjects.

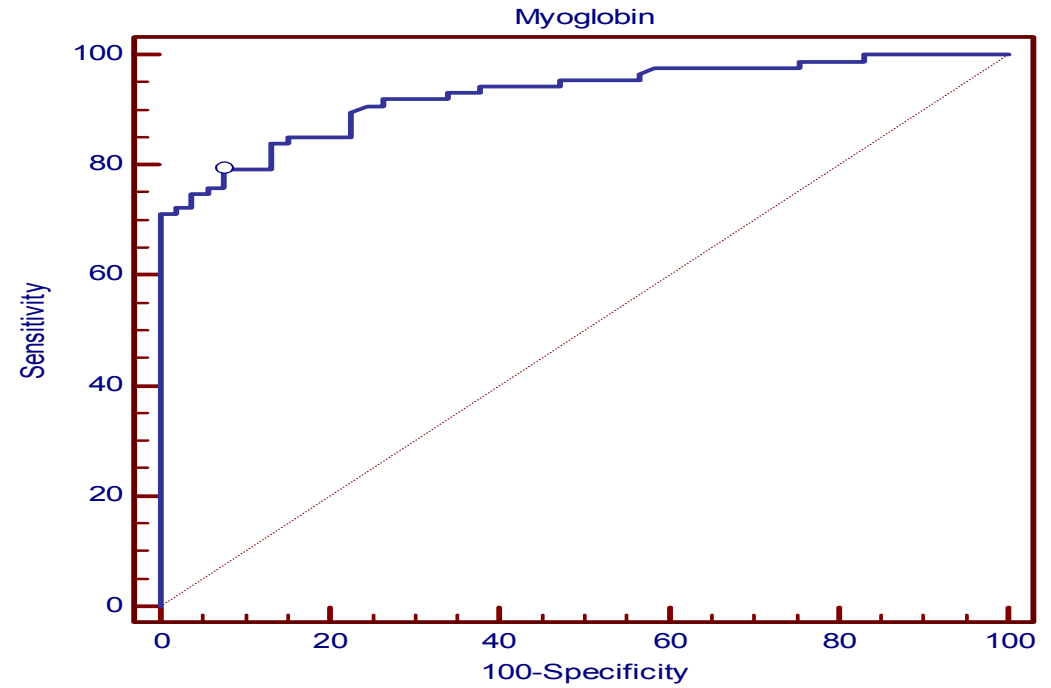

Figure3: Myoglobin ROC curve for discriminating patients who presented within 12 hours of the onset of chest pain from healthy subjects.

\section{DISCUSSION}

Taking into account the importance of the quickest possible diagnosis of acute myocardial infarction, this study attempted to assess the diagnostic value of novel cardiac biomarker $\mathrm{H}$ FABP and the performance of improved sensitivity $3^{\text {rd }}$ generation AIA-Pack assay for cTnI supplied by TOSOH. The results of this study showed that Troponin I concentration in AMI patients was significantly higher than controls (table 2) and can be used as eligible marker for discriminating patients from control since it yields a high AUC $=0.972$ with sensitivity, specificity and accuracy equal to $91.5 \%, 98 \%$ and $93.9 \%$ respectively (table
4). Furthermore, these results showed that the improved sensitivity Troponin I assay has the highest accuracy for the diagnosis of AMI patients admitted within 12 hours from the onset of chest pain when compared with H-FABP $(92.4 \%)$ and myoglobin $(83.3 \%)$. From the other hand, H-FABP showed $90.2 \%$ sensitivity and $96 \%$ specificity (table 4), these results suggested eligible role for H-FABP in the diagnosis of AMI. Different studies demonstrate high value of using this marker for the diagnosis of AMI, for example: two Japanese studies, the first by Seino et al in (2003) and the other study by Nakata et al in (2003), both of these studies showed superior performance of this 
marker over other biomarkers. Other studies that support these finding include those performed by Alhashemi in (2006), Valle et al in (2008) and Li et al in (2010). Several reasons explain the high performance of H-FABP for early diagnosis of AMI including high myocardial content, presence mainly in cytosol, low molecular weight, relative tissue specificity and early appearance in plasma and urine. Furthermore, the magnitude of the increase in plasma H-FABP may also demonstrates a good correlation with the size of infarction (Ghani et al, 2000; Cavus et al, 2006).

In contrast, some studies found that H-FABP provide limited clinical value compared with troponin I when measured in early hours after the onset of myocardial infarction like those of Alansari and Carol in (2004) and Rosman et al in (2009). This variation in the outcome of H-FABP utilization in the diagnosis of AMI is probably attributed to methodological and technical factors as well as study design and the used criteria for the diagnosis of AMI, all these factors affects the sensitivity and specificity of H-FABP (Slot et al, 2010).

Despite interest in H-FABP as an early marker of AMI diagnosis, it is still not widely used in clinical practice. One of the possible reasons of this limited use is that most of the currently available assays are not automated as the clinical application of a cardiac marker requires the availability of rapid assay kits in routine automatic systems at emergency departments (Pasaoglu et al, 2007).

Analysis of the data of this study showed that the sensitivity of myoglobin (78.1\%) and the specificity (92\%) (table 4) were lower than that of H-FABP. This could be attributed to an earlier and more rapid rise in H-FABP than in myoglobin, in addition when H-FABP compared to myoglobin, it's concentration in the heart muscle is greater than that in skeletal muscle and its normal baseline concentration is several folds lower than myoglobin. These advantages are potentially more suitable for cardiac marker than myoglobin (Alhadi and Fox, 2004).

Both H-FABP and myoglobin showed a comparable sensitivity during the two periods of time $(90.6 \%, 90 \%$ for H-FABP and $78.1 \%, 78 \%$ for myoglobin respectively), while the sensitivity of troponin I in subgroup 1 was only $(81.3 \%)$ compared with higher sensitivity in subgroup 2 (98 $\%$ ) (table 5). This increment in the sensitivity of troponin I could be attributed to the kinetic of troponin I release which may show an initial delay in release for several hours (Mad et al, 2007).

The sensitivity for diagnosing AMI cases when presented within the first 6 hours of the attack were highest with H-FABP (90.6\%) followed by troponion I (81.3\%) and myoglobin (78.1\%), these results encourage the utilization of H-FABP for those patients who are admitted within the first 6 hours from the onset of symptoms, however, the obtained sensitivity using improved troponin I assay in subgroup 1 was superior to the obtained sensitivities of troponin I which were also measured within 6 hours from the onset of symptoms in some other studies using different classical techniques, for example Zimmerman et al in (1999) using fluorometric enzyme immunoassay, Alhakim et al in (2012) using ELISA technique and Pasaoglu et al in (2007) using ELISA technique. Unlike earlier assays of troponin which has limited sensitivity within the early hours after the onset of chest pain, assays with improved sensitivity might further improve the accuracy of the diagnosis of myocardial infarction and therefore improve diagnostic sensitivity and specificity (Keller et al, 2009). In 2004, the second-generation AIA-Pack assay for cTnI from TOSOH bioscience was proved to have acceptable analytical performance for the detection of troponin I in the tested specimens (Pagani et al, 2004). More recently, the improved troponin I assay (The third-generation AIA-Pack assay for cTnI) was introduced by $\mathrm{TOSOH}$ bioscience, this assay showed an improved analytical sensitivity and reproducibility, especially at very low cTnI concentrations compared to the previous second-generation assay. (Franzini et al, 2013).

Concerning the second subgroup which included those patients who were admitted to the hospital after the $6^{\text {th }}$ hour of chest pain, up to 12 hours of chest pain, tropnin I showed the highest sensitivity equal to $(98 \%)$. Compared with $\mathrm{H}-$ FABP which showed sensitivity equal to $(90 \%)$ and myoglobin which showed sensitivity equal to $(78 \%)$, these results suggests higher performance of troponin I for those patients who presented after the $6^{\text {th }}$ hour of onset of chest pain up to 12 hours.

\section{CONCLUSIONS}

Improved sensitivity troponin I assay and $\mathrm{H}$ FABP can provide valuable information for the diagnosis of AMI. However, for those patients who present within the first 6 hours of the onset of chest pain, H-FABP will provide superior sensitivity, as an early biomarker that potentially reduces diagnostic uncertainty for patients with suspected AMI and allow an earlier initiation of treatment, while for those patients who presented later after the $6^{\text {th }}$ hour up to 12 hours of the onset of chest pain, it is more appropriate to use troponin I assay as it provide higher sensitivity. 


\section{REFERENCES}

Achar S.; Kundu S.; Norcross W. (2005, July). Diagnosis of acute coronary syndrome. $\mathrm{Am}$ Fam Physician. 72. P.p 119-126.

Alansari S.; Croal B. (2004, September). Diagnostic value of heart fatty acid binding protein and myoglobin in patients admitted with chest pain. Ann Clin Biochem. 41. P.p 391-396.

Alhadi H.; Fox K. (2004, April). Do we need additional markers of myocyte necrosis: the potential value of heart fatty -acid binding protein. QJ Med. 97. P.p187-198.

Alhakim D, Al-Quobaili F, Shahin E (2012). Comparison of serum H-FABP, cTnI and $C K-M B$ levels in myocardial infarction. Retrieved from: http://scla.org.sy/magazine /issues/6 4/423.html on November 2013.

Alhashemi J (2006, March). Diagnostic accuracy of a bedside qualitative immunochromatographic test for acute myocardial infarction. Am J Emerg Med. 24. P.p 149-155.

Al-Mukhtar S.; Ahmad A. (2011). Comparison between quantitative and qualitative biochemical markers in the diagnosis of acute coronary syndrome. Irq J Pharm. 11(2). P.p 101-109.

Cavus U.; Coskun F.; Yavus B.; et al. (2006, July). Heart - type fatty acid binding protein can be a diagnostic marker in acute coronary syndrome. J Natl Med.98(7). P1067.

Chan D.; Ng L. (2010, June) Biomarkers in acute myocardial infarction. $B M C$ Med. 8. P.p. $34-45$.

Das M (2012). Biomarkers in acute coronary syndrome, heart failure and acute kidney injury. Satellites. 22. P.p.1-6.

Eggers K. (2007). Cardiac troponins in patients with suspected or confirmed acute coronary syndrome. Ph.D. thesis, Faculty of Medicine, Uppsala University, Sweden.

Erhardt L.; Herlitz J.; Bossaert L.; et al. (2002 August). Task force on the management of chest pain. Eur Heart J. 23. P.p.1153-1176.

Franzini M.; Prontera C.; Masotti S.; et al. (2013, February). Evaluation of analytical performance of a novel immunoenzymometric assay for cTnI. Clin Chim Acta. 416. P.p. 48-49/Abstract.

Ghani F.; Wu A.; Graff L.; et al. (2000, May). Role of heart- type fatty acid binding protein in early detection of acute myocardial infarction. Clin Chem. 46 (4). P.p.718-719.

Jaffe A.; Ravkilde J.; Roberts R.; et al. (2000, September). It's time for a change to a troponin standard. Circulation. 102. P.p. 1216-1220.

Keller T.; Zeller T.; Peelz D.; et al. (2009, August). Sensitive Troponin I Assay in Early Diagnosis of Acute Myocardial Infarction. N Engl J Med. 361. P.p. 868-877.

Kumar A.; Cannon C. (2009, October). Acute coronary syndrome: diagnosis and management, part I. Mayo Clin Proc. 84(10). P.p. 917-938.

Li C.; Li J.; Liang X.; et al. (2010, March). Point of care test of heart type fatty acid binding protein for the diagnosis of early acute myocardial infarction. Acta Pharmacol Sin. 31. P.p. 307-312.

Lin S.; Yokoyama H.; Rac V.; et al. (2012, June). Novel biomarkers in diagnosing cardiac ischemia in the emergency department: a systemic review. Resuscitation. 83. P.p. 684691.

Mad P.; Domanovits H.; Fazelnia C.; et al. (2007, March). Human heart -type fatty binding protein as a point of care test in the early diagnosis of acute myocardial infarction. $Q J$ Med. 100. P.p. 203-210.

Melanson S.; Tanasijevic M.; Jarolim P. (2007, October). Cardiac troponin assays: a view from the clinical chemistry laboratory. Circulation. 116. P.p. e501-e504.

Nakata T.; Hashimoto A.; Hase M.; et al. (2003). Human heart-type fatty acid-binding protein as an early diagnostic and prognostic marker in acute coronary syndrome. Cardiology. 99. P.p. 96-104/Abstract.

Owens P.; Barrett M.; Gibson T.; et al. (2010, August). Emergency department care in the United States: a profile of national data sources. Ann Emerg Med. 56. P.p.150-165.

Pagani F.; Stefini F.; Gianmatteom.; et al. (2004, September). Multicenter evaluation of the TOSOH AIA-pack second generation cardiac troponin I assay. Clin Chem. 50 (9). P.p. 1707-1709.

Pasaoglu H.; Ofluoglu E.; Ilhan M.; et al. (2007). The role of Heart -type fatty acid binding protein (H-FABP) in acute myocardial infarction (AMI) compared to conventional cardiac biochemical markers. Turk $J$ Med Sci. 37(2). P.p. 61-67.

Paterson D.; Herring N. (2006, April). ECG diagnosis of acute ischemia and infarction: past, present and future. $Q J$ Med. 99. P.p 219-230.

Reiter M.; Twerenbold R.; Reichlin T.; et al. (2011, June). Early diagnosis of acute myocardial infarction in the elderly using 
more sensitive cardiac troponin assays. Eur Heart J. 32. P.p. 1379-1389.

Rosman J.; Kavala G.; Obunai K.; et al. (2009, Summer). The role of heart-type fatty acidbinding protein in the diagnosis of acute coronary syndrome. Int J Angiol. 18(2). P.p. 79-81.

Seino Y.; Ogata K.; Takano T.; et al. (2003, August). Use of a whole blood rapid panel test for heart-type fatty acid-binding protein in patients with acute chest pain: comparison with rapid troponin $\mathrm{T}$ and myoglobin tests. Am J Med. 115 (3). P.p 185-190.

Slot M.; Reitsma J.; Rutten F.; et al. (2010, December). Heart-type fatty acid-binding protein in the early diagnosis of acute myocardial infarction: a systemic review and meta-analysis. Heart. 96. P.p.1957-1963.

Valle H.; Riesgo L.; Bel M.; et al. (2008, June). Clinical assessment of heart-type fatty acid binding protein in early diagnosis of acute coronary syndrome. Eur J Emerg Med.15. P.p. 140-144.

Zimmerman J.; Fromm R.; Meyer D.; et al. (1999, April). Diagnostic marker cooperative study for the diagnosis of myocardial infarction. Circulation. 99. P.p.1671-1677. 